Journal of Mathematics and Statistics 8 (2): 241-247, 2012

ISSN 1549-3644

(C) 2012 Science Publications

\title{
Foldings and Deformation Retract of Hyperhelix
}

\author{
${ }^{1}$ A.E. El-Ahmady and ${ }^{1,2}$ E. AL-Hesiny \\ ${ }^{1,2}$ Department of Mathematics, \\ Faculty of Science, Taibah University, Madinah, Saudi Arabia \\ ${ }^{1}$ Department of Mathematics, Faculty of Science, Tanta University, Tanta, Egypt
}

\begin{abstract}
Our aim in the present study is to introduce and study new types of retractions of hyperhelix in Minkowski space. Types of the deformation retracts of hyperhelix in Minkowski space were discussed. The relations between the foldings and the deformation retracts of hyperhelix in Minkowski space were deduced. Types of minimal retractions of hyperhelix in Minkowski space were obtained. Also, the connection between retractions and T, N, B, K and $\tau$, of hyperhelix in Minkowski space were presented. New types of the minimal retractions and the end of the limits of foldings of hyperhelix in Minkowski space are deduced.
\end{abstract}

Key words: Hyperhelix in minkowski space, folding, minimal retractions, deformation retracts of hyperhelix

\section{INTRODUCTION}

An $\mathrm{n}$-dimensional topological manifold $\mathrm{M}$ is $\mathrm{a}$ Hausdorff topological space with a countable basis for the topology which is locally homeomorphic to $\mathbb{R}^{n}$. If $\mathrm{h}$ : $\mathrm{U} \rightarrow \mathrm{U}^{\prime}$ is a homeomorphism of $\mathrm{U} \subseteq \mathrm{M}$ onto $\mathrm{U} \subseteq \mathbb{R}^{n}$, then $\mathrm{h}$ is called a chart of $\mathrm{M}$ and $\mathrm{U}$ is the associated chart domain. A collection $\mathrm{h}_{\alpha}, \mathrm{U}_{\alpha}$ is said to be an atlas for $\mathrm{M}$ if $U_{\alpha \in A} U_{\alpha}=M$. Given two charts $h_{\alpha}$ and $h_{\beta}$ such that $\mathrm{U}_{\alpha \beta}=\mathrm{U}_{\alpha} \cap \mathrm{U}_{\beta} \neq \varnothing$, the transformation chart $\mathrm{h}_{\beta} \mathrm{O} \mathrm{h}_{\alpha}{ }^{-1}$ between open sets of $\mathbb{R}^{n}$ is defined and if all of these charts transformation are $\mathrm{C}^{\infty}$-mappings, then the manifolds under consideration is a $\mathrm{C}^{\infty}$-manifolds. A differentiable structure on $\mathrm{M}$ is a differentiable atlas and a differentiable manifold is a topological manifold with a differentiable structure (Catoni et al., 2008; Naber, 2011a; 2011b; Reid and Szendroi. 2005; Lopez, 2008; Shick, 2007).

Most folding problems are attractive from a pure mathematical standpoint, for the beauty of the problems themselves. The folding problems have close connections to important industrial applications. Linkage folding has applications in robotics and hydraulic tube bending. Paper folding has applied in sheet-metal bending, packaging and air-bag folding (Demainel, 2001). Isometric folding between two Riemannian manifolds may be characterized as maps that send piecewise geodesic segments to a piecewise geodesic segment of the same length (ElAhmady, 2007a; El-Ahmady and Rafat, 2006; DIFrancesco, 2000). For a topological folding the maps does not preserve lengths (El-Ahmady, 2004a; 2004b; 2011; El-Ahmady and Al-Hesiny, 2011). A subset A of a topological space $\mathrm{X}$ is called a retract of $\mathrm{X}$ if there exists a continuous map $\mathrm{r}: \mathrm{X} \longrightarrow \mathrm{A}$ such that $\mathrm{r}(\mathrm{a})=\mathrm{a} \forall \mathrm{a} \in \mathrm{A}$, where $\mathrm{A}$ is closed and $\mathrm{X}$ is open (ElAhmady, 2006; 1994; 2011; Michael, 2003; Baronti et al., 2003; Pellicer-Covarrubias, 2004). Also, a subset A of a topological space $\mathrm{X}$ is a deformation retracts of $\mathrm{X}$ if there exists a retraction $\mathrm{r}: \mathrm{X} \longrightarrow \mathrm{A}$ and a homotopy $\varphi: \mathrm{X} \times \mathrm{I} \longrightarrow \mathrm{X}$ such that:

$$
\begin{aligned}
& \left.\begin{array}{l}
\varphi(x, 0)=x \\
\varphi(x, 1)=r(x)
\end{array}\right\} x \in X \\
& \varphi(a, t)=a, a \in A, t \in[0,1]
\end{aligned}
$$

(El-Ahmady, 2004b; El-Ahmady and Shamara, 2001; El-Ahmady, 2011b; Naber, 2011b; Reid and Szendroi, 2005). The helix is one of the most fascinating curves in science and nature. From the view of differential geometry, a helix is a geometrical curve with nonvanishing constant curvature (or first curvature of the curve and denoted by $\mathrm{K}_{1}$ ) and non-vanishing constant torsion (or second curvature of the curve and denoted by $\mathrm{K}_{2}$. A curve of constant slope or general helix in Euclidean 3 -space $\mathrm{E}^{3}$, is defined by the property that the tangent makes a constant angle with a fixed straight line (Yaliniz and Hacisalihoglu, 2007; Walrave, 1995; Kocayigit and Onder, 2007; Ilarslan and Boyacioglu, 2008).

The aim of this study is to describe the hyperhelix in Minkowski space geometrically, specifically concerned 
with the study of the new types of retraction, deformation retract and the folding of hyperhelix in Minkowski space from viewpoint of the variation of the density function on chaotic spheres in chaotic space-like Minkowski space time, folding of fuzzyhypertori and their retractions, limits of fuzzy retractions of fuzzy hyperspheres and their foldings, fuzzy folding of fuzzy horocycle, fuzzy Lobachevski a space and its folding. The deformation retract and topological folding of Buchdahi space, retraction of chaotic Ricci space, a calculation of geodesics in chaotic flat space and its folding, fuzzy deformation retract of fuzzy horospheres, on fuzzy spheres in fuzzy Minkowski space, retraction of chaotic black hole, the topological folding of the hyperbola in Minkowski 3-space and The geodesic deformation retract of Klein Bottle and its folding as presented by (ElAhmady, 2007a; 2007b; El-Ahmady and Rafat, 2006; ElAhmady, 2004a; 2004b; El-Ahmady, 1994; El-Ahmady and Rafat, 2009; El-Ahmady and Shamara, 2001; ElAhmady and El-Araby, 2010; El-Ahmady, 2011a; 2011b; El-Ahmady and Al-Hesiny, 2011).

Main results: Now, we introduce types of retractions of the open helix $\left\{(\right.$ accost, asint, bt $-\sigma\}=\mathrm{H}^{1}$ with non 0-curvature and its velocity is:

$$
\begin{gathered}
\mathrm{H}^{1}(\mathrm{t})=(\text {-asint, acost, } \mathrm{b}) \neq 0, \mathrm{H}^{1}=(\text {-acost, }- \text { asint }, 0), \\
\mathrm{T}=\frac{1}{\sqrt{\mathrm{a}^{2}-\mathrm{b}^{2}}}(\text {-asint, acost, } \mathrm{b}), \mathrm{N}=(-\cos t,-\sin \mathrm{t}, 0), \\
\mathrm{B}=\frac{1}{\sqrt{\mathrm{a}^{2}-\mathrm{b}^{2}}}(-\mathrm{b} \sin \mathrm{t}, \mathrm{b} \cos \mathrm{t}, \mathrm{a}), \mathrm{K}=\frac{\mathrm{a}}{\mathrm{a}^{2}-\mathrm{b}^{2}} \text { and } \tau=\frac{-\mathrm{b}}{\mathrm{a}^{2}-\mathrm{b}^{2}} .
\end{gathered}
$$

Since $\left(H^{-1}(t), H^{-1}(t)\right)=a^{2}-b^{2}$, then this helix is a space like curve if $a^{2}>b^{2}$, a time like curve if $a^{2}>b^{2}$ and a null like curve if $\mathrm{a}^{2}=\mathrm{b}^{2 .}$ Let $\mathrm{r}_{\mathrm{i}}: \mathrm{H}^{1} \rightarrow \overline{\mathrm{H}^{1}}, \overline{\mathrm{H}^{1}} \subset \mathrm{H}^{1}$ be the retraction map of $\mathrm{H}^{1}$ such that: $\mathrm{r}_{1}\left(\mathrm{H}^{1}\right)=(\mathrm{a}, 0,0)$, $\mathrm{t}=$ 0 . In this case $\mathrm{r}_{1}{ }_{1}\left(\mathrm{H}^{1}\right)=0$ and $\left(\mathrm{r}_{1}{ }_{1}\left(\mathrm{H}^{1}\right), \mathrm{r}^{\circ}\left(\mathrm{H}^{1}\right)\right)=0$ then this retraction is a space like curve:

$$
\begin{aligned}
& \mathrm{r}_{2}\left(\mathrm{H}^{1}\right)=\left(\frac{\sqrt{3}}{2} \mathrm{a} \frac{1}{2} \mathrm{a} \frac{\pi}{6} \mathrm{~b}\right), \mathrm{t}=\frac{\pi}{6}, \mathrm{r}_{3}\left(\mathrm{H}^{1}\right)=\left(\frac{\sqrt{2}}{2} \mathrm{a} \frac{\sqrt{2}}{2} \mathrm{a} \frac{\pi}{4} \mathrm{~b}\right), \mathrm{t}=\frac{\pi}{4}, \\
& \mathrm{r}_{4}\left(\mathrm{H}^{1}\right)=\left(\frac{1}{2} \mathrm{a}, \frac{\sqrt{3}}{2} \mathrm{a}, \frac{\pi}{3} \mathrm{~b}\right), \mathrm{t}=\frac{\pi}{3}, \mathrm{r}_{5}\left(\mathrm{H}^{1}\right)=\left(0, \mathrm{a}, \frac{\pi}{2} \mathrm{~b}\right), \mathrm{t}=\frac{\pi}{2}, \\
& \mathrm{r}_{6}\left(\mathrm{H}^{1}\right)=\left(\frac{-1}{2} \mathrm{a}, \frac{\sqrt{3}}{2} \mathrm{a}, \frac{\pi}{3} \mathrm{~b}\right), \mathrm{t}=\frac{2 \pi}{3} . \text { In this case } \mathrm{r}_{6}^{\prime} \\
& \left(\mathrm{H}^{1}\right)=0 \text { and }\left(\mathrm{r}_{6}\left(\mathrm{H}^{1}\right) \mathrm{r}_{6}\left(\mathrm{H}^{1}\right)\right)=0 \text { then this retraction is } \mathrm{a}
\end{aligned}
$$

space like curve. $r_{7}\left(H^{1}\right)=\left(\frac{-\sqrt{2}}{2} a, \frac{\sqrt{2}}{2} a, \frac{3 \pi}{4} b\right), t=\frac{3 \pi}{4}$. In this retraction $\mathrm{r}_{7}\left(\mathrm{H}^{1}\right)=0$ and $\left.\hat{r}_{7}\left(\mathrm{H}^{1}\right)\right)=0$ then this retraction is a space like curve. $\mathrm{r}_{8}\left(\mathrm{H}^{1}\right)=$ $\left(\frac{-\sqrt{3}}{2} \mathrm{a}, \frac{1}{2} \mathrm{a}, \frac{5 \pi}{6} \mathrm{~b}\right), \quad \mathrm{t}=\frac{5 \pi}{6}, \mathrm{r}_{9}\left(\mathrm{H}^{1}\right)=(-\mathrm{a}, 0, \pi \mathrm{b}), \mathrm{t}=\pi$. In this retraction $\hat{r}_{9}\left(H^{1}\right)=0$ and $\left(\hat{r}_{9}\left(H^{1}\right), \hat{r}_{9}\left(H^{1}\right)\right)$ $=0$, then this retraction is a space like curve:

$$
\begin{gathered}
\mathrm{r}_{10}\left(\mathrm{H}^{1}\right)=\left(\frac{-\sqrt{3}}{2} \mathrm{a}, \frac{-1}{2} \mathrm{a}, \frac{7 \pi}{6} \mathrm{~b}\right), \mathrm{t}=\frac{7 \pi}{6}, \mathrm{r}_{11}\left(\mathrm{H}^{1}\right)=\left(\frac{-\sqrt{2}}{2} \mathrm{a}, \frac{-\sqrt{2}}{2} \mathrm{a}, \frac{5 \pi}{4} \mathrm{~b}\right), \\
\mathrm{t}=\frac{5 \pi}{4}, \mathrm{r}_{12}\left(\mathrm{H}^{1}\right)=\left(\frac{-1}{2} \mathrm{a}, \frac{-\sqrt{3}}{2} \mathrm{a}, \frac{4 \pi}{3} \mathrm{~b}\right), \mathrm{t}=\frac{4 \pi}{3}, \mathrm{r}_{13}\left(\mathrm{H}^{1}\right) \\
=\left(0,-\mathrm{a}, \frac{3 \pi}{2} \mathrm{~b}\right), \mathrm{t}=\frac{3 \pi}{2}, \mathrm{r}_{14}\left(\mathrm{H}^{1}\right)=\left(\frac{1}{2} \mathrm{a}, \frac{-\sqrt{3}}{2} \mathrm{a} \frac{5 \pi}{3} \mathrm{~b}\right), \mathrm{t}=\frac{5 \pi}{3}, \\
\mathrm{r}=\frac{-\pi}{3}, \mathrm{r}_{16}\left(\mathrm{H}^{1}\right)=\left(\frac{\sqrt{2}}{2} \mathrm{a}, \frac{-\sqrt{2}}{2} \mathrm{a}, \frac{7 \pi}{4} \mathrm{~b}\right), \\
\mathrm{t}=\frac{7 \pi}{4}, \mathrm{r}_{17}\left(\mathrm{H}^{1}\right)=\left(\frac{1}{2} \mathrm{a}, \frac{-\sqrt{3}}{2} \mathrm{a}, \frac{-\pi}{3} \mathrm{~b}, \frac{-\sqrt{2}}{2} \mathrm{a} \frac{-\pi}{4} \mathrm{~b}\right), \mathrm{t}=\frac{-\pi}{4}, \\
\mathrm{r}_{18}\left(\mathrm{H}^{\mathrm{l}}\right)=\left(\frac{-\pi}{2} \mathrm{a}, \frac{-1}{2} \mathrm{a}, \frac{11 \pi}{6} \mathrm{~b}\right), \mathrm{t}=\frac{11 \pi}{6}, \mathrm{r}_{19}\left(\mathrm{H}^{\mathrm{l}}\right)=\left(\frac{\sqrt{3}}{2} \mathrm{a}, \frac{-1}{2} \mathrm{a}, \frac{-\pi}{6} \mathrm{~b}\right), \\
\mathrm{r}_{20}\left(\mathrm{H}^{1}\right)=\left(-0.1423 \mathrm{a}, 0.9898 \mathrm{a}, \frac{6 \pi}{11}\right) . \mathrm{In}
\end{gathered}
$$

this case $\mathbf{r}_{20}\left(\mathrm{H}^{1}\right)=0$ and $\left(\mathrm{r}_{20}{ }_{20}\left(\mathrm{H}^{1}\right) \mathrm{r}_{20}\left(\mathrm{H}^{1}\right)\right)=0$, then this retraction is a space like curve.

Hence, we can formulate the following theorems:

Theorem 1: Let $r\left(\mathrm{H}^{1}\right)$ be the retraction map of the helix $\mathrm{H}^{1} \subset \mathrm{E}_{1}{ }^{3}$. If $\operatorname{dim} \mathrm{r}\left(\mathrm{H}^{1}\right)=0$, then $\mathrm{T}, \mathrm{N}, \mathrm{B}, \mathrm{K}$ and $\tau$ of the retraction of the helix are unlimited.

Theorem 2: Let $r\left(\mathrm{H}^{1}\right)$ be the retraction map of the helix $\mathrm{H}^{1} \subset \mathrm{E}_{1}{ }^{3}$. If $\operatorname{dim} \mathrm{r}\left(\mathrm{H}^{1}\right)=0$, then this retraction of the helix is a space like curve.

Theorem 3: Under the retraction map a spacelike helix $\mathrm{H}^{1} \subset \mathrm{E}_{1}^{3}$ has curvature identically zero if and only if $\mathrm{r}$ $\left(\mathrm{H}^{1}\right) \subset \mathrm{E}_{1}^{3}$ is a part of a straight line. 
Theorem 4: Under the retraction map a space like helix $\mathrm{H}^{1} \subset \mathrm{E}_{1}{ }^{3}$ has torsion identically zero if and only if $\mathrm{r}\left(\mathrm{H}^{1}\right)$ $\subset \mathrm{E}_{1}{ }^{3}$ is a planar curve.

In this position, we present new types of retractions which preserve the dimension given by: $r_{21}\left(H^{1}\right)=$ (acost*, asint*, bt*), $0<\mathrm{t}^{*}<\mathrm{n} \mathrm{n} \in \mathbb{N}$. In this retraction $\mathrm{r}_{21}\left(\mathrm{H}^{1}\right)=\left(-\right.$ asint*, accost*, b) and $\left(\mathrm{r}^{\prime}{ }_{21}\left(\mathrm{H}^{1}\right), \mathrm{r}_{21}{ }_{21}\left(\mathrm{H}^{1}\right)\right)=$ $a^{2}-b^{2}$, then this retraction is a space like curve if $a^{2}>b^{2}$, a time like curve if $a^{2}<b^{2}$, or a null like curve if $a^{2}=b^{2}$.

$$
\begin{aligned}
& \mathrm{r}_{22}\left(\mathrm{H}^{1}\right)=\left(\operatorname{acost}^{*}, \operatorname{asint}^{*}, \mathrm{bt}^{*}\right),-\mathrm{n}<\mathrm{t}^{*} \leq 0, \mathrm{n} \in \mathbb{N}, \\
& \mathrm{r}_{23}\left(\mathrm{H}^{1}\right)=\left(\operatorname{acost}{ }^{*}, \operatorname{asint} \mathrm{t}^{*}, \mathrm{bt} *\right), \mathrm{c}<\mathrm{t}^{*} \leq \mathrm{c}^{2}, \mathrm{c} \in \mathbb{R}, \\
& \mathrm{r}_{24}\left(\mathrm{H}^{1}\right)=\left(\operatorname{acost} t^{*}, \operatorname{asint}^{*}, \mathrm{bt} *\right), \frac{\mathrm{c}}{\mathrm{n}}<\mathrm{t}^{*}<\mathrm{c}, \mathrm{c} \in \mathbb{R}, \mathrm{n} \in \mathbb{N}, \\
& \mathrm{r}_{25}\left(\mathrm{H}^{1}\right)=\left(\text { acost* } \mathrm{asint}^{*}, \mathrm{bt} *\right),-\mathrm{n} \leq \mathrm{t}^{*}<\mathrm{n}, \mathrm{n} \in \mathbb{N}, \\
& \mathrm{r}_{26}\left(\mathrm{H}^{1}\right)=\left(\operatorname{acost}{ }^{*}, \operatorname{asint} *, \mathrm{bt} *\right), \sqrt{|\mathrm{c}|} \leq \mathrm{t}^{*} \leq|\mathrm{c}|, \mathrm{c} \in \mathbb{R} \\
& r_{27}\left(H^{1}\right)=(\text { acost, } \operatorname{asin}|t|, b|t|), \\
& \mathrm{r}_{28}\left(\mathrm{H}^{1}\right)=\left(\operatorname{acost} \mathrm{t}^{*}, \operatorname{asint}^{*}, \mathrm{bt} *\right), \mathrm{c}<\mathrm{t}^{*}<\mathrm{d}, \mathrm{c}, \mathrm{d} \in \mathbb{R}, \mathrm{c}<\mathrm{d}, \\
& r_{29}\left(H^{1}\right)=(\text { acost, asint, }|b t|) .
\end{aligned}
$$

This leads to the following theorems:

Theorem 5: Under the retraction map a spacelike helix $\mathrm{H}^{1} \subset \mathrm{E}_{1}^{3}$ has torsion identically zero and curvature is bigger than zero if and only if $r\left(\mathrm{H}^{1}\right) \subset$ $\mathrm{E}_{1}^{3}$ is a part of a circle.

Theorem 6: Let $r\left(\mathrm{H}^{1}\right)$ is the retraction map of the helix $\mathrm{H}^{1} \subset \mathrm{E}_{1}^{3}$. If $\operatorname{dim} \mathrm{r}\left(\mathrm{H}^{1}\right)=\operatorname{dim} \mathrm{H}^{1}$, then $\mathrm{T}, \mathrm{N}, \mathrm{B}$, $\mathrm{K}$ and $\tau$ of $\mathrm{r}\left(\mathrm{H}^{1}\right)$ are the same as or different from $\mathrm{T}$, $\mathrm{N}, \mathrm{B}, \mathrm{K}$ and $\tau$ of $\mathrm{H}^{1}$.

Theorem 7: Let $\mathrm{r}\left(\mathrm{H}^{1}\right)$ be the retraction map of the helix $\mathrm{H}^{1} \subset \mathrm{E}_{1}^{3}$. If $\operatorname{dim} \mathrm{r}\left(\mathrm{H}^{1}\right)=\operatorname{dim} \mathrm{H}^{1}$, then this retraction of the helix is a space like curve if $a^{2}>b^{2}, a$ time like curve if $a^{2}<b^{2}$, or a null like curve if $a^{2}=b^{2}$.

Theorem 8: If the deformation retract of the helix $\mathrm{H}^{1} \subset$ $\mathrm{E}_{1}^{3}$ is $\mathrm{D}: \mathrm{H}^{1} \times \mathrm{I} \rightarrow \mathrm{H}^{1}$, where $\mathrm{I}$ is the closed interval $[0,1]$, the retraction of $\mathrm{H}^{1} \subset \mathrm{E}_{1}^{3}$ is $\mathrm{r}: \mathrm{H}^{1} \rightarrow \mathrm{H}^{* 1}, \mathrm{H}^{* 1} \subset \mathrm{H}^{1}$ and the folding of $\mathrm{H}^{1}$ into itself is $\mathrm{f}: \mathrm{H}^{1} \rightarrow \mathrm{H}^{1}$. Then there induce deformation retract, retractions and foldings such that the following diagram is commutative.

Proof: Let the deformation retract of $\mathrm{H}^{1} \subset \mathrm{E}_{1}^{3}$ is $\mathrm{D}_{1}$ : $\mathrm{H}^{1} \times \mathrm{I} \rightarrow \mathrm{H}^{1}$, the folding of $\mathrm{H}^{1} \times \mathrm{I}$ and $\mathrm{D}_{1}\left(\mathrm{H}^{1} \times \mathrm{I}\right)$ are defined by $f_{1}:\left(\mathrm{H}^{1} \times \mathrm{I}\right) \rightarrow \mathrm{H}^{1} \times \mathrm{I}$ and $f_{2}: \mathrm{D}_{1}\left(\mathrm{H}^{1} \times \mathrm{I}\right) \rightarrow \mathrm{H}^{1}$,

$\mathrm{D}_{2}: f_{1}\left(\mathrm{H}^{1} \times \mathrm{I}\right) \rightarrow \mathrm{H}^{1}$ and the retractions of $\mathrm{D}_{2}\left(f_{1}\left(\mathrm{H}^{1} \times \mathrm{I}\right)\right)$ and $f_{2}\left(\mathrm{D}_{1}\left(\mathrm{H}^{1} \times \mathrm{I}\right)\right)$ are given by $\mathrm{r}_{1}: \mathrm{D}_{2}\left(f_{1}\left(\mathrm{H}^{1} \times \mathrm{I}\right)\right) \rightarrow \mathrm{H}^{0}$ and $\mathrm{r}_{2}: f_{2}\left(\left(\mathrm{D}_{1}\left(\mathrm{H}^{1} \times \mathrm{I}\right)\right)\right) \rightarrow \mathrm{H}^{0}, \mathrm{H}^{0}$ is a 0 -dimensional space. Hence, the following diagram is commutative:

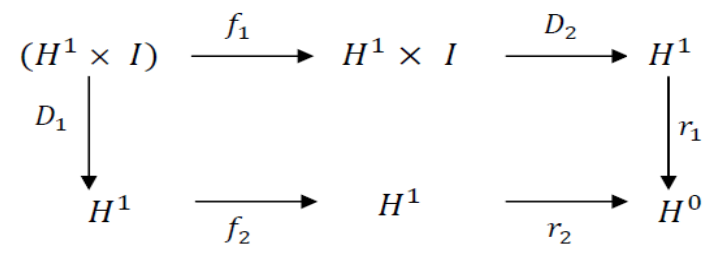

i.e., $r_{1} \circ D_{2}$ of $f_{1}\left(H^{1} \times I\right)=r_{2} \circ f_{2} D_{1}\left(H^{1} \times I\right)$

Theorem 9: Let $\mathrm{H}^{1} \subset \mathrm{E}_{1}^{3}$ be the helix in 3Minkowski space, then the relation between the retraction $\mathrm{r}: \mathrm{H}^{1} \rightarrow \mathrm{H}^{* 1}, \mathrm{H}^{* 1} \subset \mathrm{H}^{1}$ and the limit of the foldings $\lim _{\mathrm{m} \rightarrow \infty} f_{\mathrm{m}}: \mathrm{H}^{1} \rightarrow \mathrm{H}^{0}$ discussed from the following commutative diagram.

Proof: Let the retraction of helix $\mathrm{r}_{1}: \mathrm{H}^{1} \rightarrow \mathrm{H}^{* 1}$, the limit of the foldings of helix $\mathrm{H}^{1}$ is $\lim _{\mathrm{m} \rightarrow \infty} f_{\mathrm{m}}$ : $\mathrm{H}^{1} \rightarrow \mathrm{H}^{0}, \lim _{\mathrm{m} \rightarrow \infty} f_{\mathrm{m}+1}: \mathrm{r}_{1}\left(\mathrm{H}^{1}\right) \rightarrow \mathrm{H}^{0}$ and $\mathrm{r}_{2}: \lim _{\mathrm{m} \rightarrow \infty} f_{\mathrm{m}}$ $\left(\mathrm{H}^{1}\right) \rightarrow \mathrm{H}^{0}, \mathrm{H}^{0}$ is a 0 -dimensional space, then the following diagram is commutative:

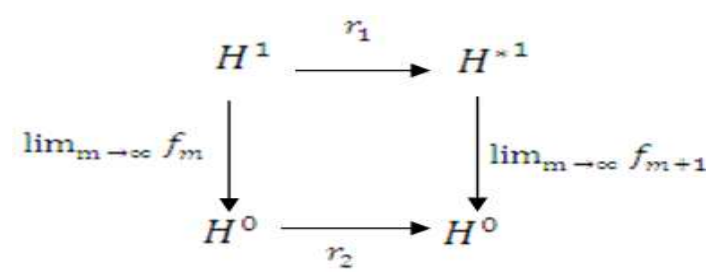

i.e., $\lim _{\mathrm{m} \rightarrow \infty} f_{\mathrm{m}+1}$ o $\mathrm{r}_{1}\left(\mathrm{H}^{1}\right)=\mathrm{r}_{2}$ o $\lim _{\mathrm{m} \rightarrow \infty} f_{\mathrm{m}}\left(\mathrm{H}^{1}\right)$.

Theorem 10: The end of the limits of folding of $\mathrm{H}^{1}$ into itself coincides with the minimal retractions.

Proof: Let $r_{i}$ be the retractions, $f_{\mathrm{i}}$ are the foldings and $\sigma_{\mathrm{I}}$ are the homeomorphisms. Then:

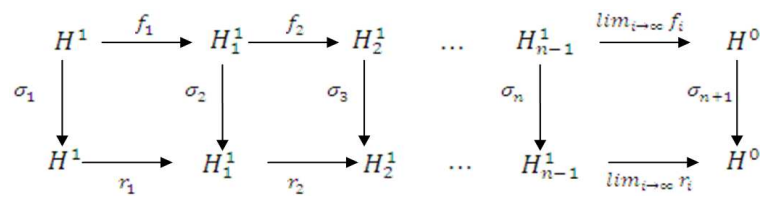


Theorem 11: Given a deformation retract D: $H^{1} \times I$ $\rightarrow \mathrm{H}^{1}$, retraction $\mathrm{r}: \mathrm{H}^{1} \times \mathrm{I} \rightarrow \mathrm{H}^{* 1}, \mathrm{H}^{* 1} \subset \mathrm{H}^{1}$ and the limit of the folding is $\lim _{\mathrm{m} \rightarrow \infty} f_{\mathrm{m}}: \mathrm{H}^{\times 1} \rightarrow \mathrm{H}^{0}$, then $\lim _{\mathrm{m} \rightarrow \infty} f_{\mathrm{m}}$ or $_{1}$ $\left(\mathrm{H}^{1} \times \mathrm{I}\right)=\mathrm{r}_{2} \circ \mathrm{D}\left(\mathrm{H}^{1} \times \mathrm{I}\right)$.

Proof: Let the retraction $\mathrm{r}_{1}: \mathrm{H}^{1} \times \mathrm{I} \rightarrow \mathrm{H}^{* 1}$, the deformation retract of helix is $\mathrm{D}: \mathrm{H}^{1} \times \mathrm{I} \rightarrow \mathrm{H}^{1}$, the retraction of $\mathrm{D}\left(\mathrm{H}^{1} \times \mathrm{I}\right)$ is $\mathrm{r}_{2}$ : $\mathrm{D}\left(\mathrm{H}^{1} \times \mathrm{I}\right) \rightarrow \mathrm{H}^{0}$ and the limit of the folding of $\mathrm{r}_{1}\left(\mathrm{H}^{1} \times \mathrm{I}\right)$ is $\lim _{\mathrm{m} \rightarrow \infty} f_{\mathrm{m}}: \mathrm{r}_{1}\left(\mathrm{H}^{1} \times \mathrm{I}\right) \rightarrow \mathrm{H}^{0}$. Then from the following diagram, we have:

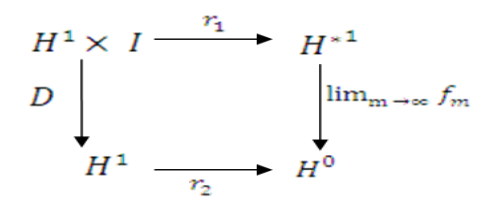

$$
\lim _{m \rightarrow \infty} f_{m} o r_{1}\left(H^{1} \times I\right)=r_{2} o D\left(H^{1} \times I\right) .
$$

Now, consider the open hyperhelix in Minkowski 4-space $\mathrm{H}^{2} \subset \mathrm{E}_{1}^{4}$ defined as:

$$
\begin{aligned}
& \left\{\left(\mathrm{a} \cos \left(\frac{\mathrm{r}}{\sqrt{\mathrm{a}^{2} \mathrm{r}^{2}+\mathrm{b}^{2}}} \mathrm{t}\right), \mathrm{a} \sin \left(\frac{\mathrm{r}}{\sqrt{\mathrm{a}^{2} \mathrm{r}^{2}+\mathrm{b}^{2}}} \mathrm{t}\right),\right.\right. \\
& \left.\left.\mathrm{b} \cos \left(\frac{1}{\sqrt{\mathrm{a}^{2} \mathrm{r}^{2}+\mathrm{b}^{2}}} \mathrm{t}\right), \mathrm{b} \sin \left(\frac{1}{\sqrt{\mathrm{a}^{2} \mathrm{r}^{2}+\mathrm{b}^{2}}} \mathrm{t}\right)\right)-\sigma\right\}=\mathrm{H}^{2}, \sigma
\end{aligned}
$$

be a point in hyperhelix in Minkowski 4-space. The velocity is:

$$
\begin{aligned}
& H^{2}(t)=\left(-a \frac{r}{\sqrt{a^{2} r^{2}+b^{2}}} \sin \left(\frac{r}{\sqrt{a^{2} r^{2}+b^{2}}} t\right),\right. \\
& a \frac{r}{\sqrt{a^{2} r^{2}+b^{2}}} \cos \left(\frac{r}{\sqrt{a^{2} r^{2}+b^{2}}} t\right),-b \frac{1}{\sqrt{a^{2} r^{2}+b^{2}}} \\
& \left.\sin \left(\frac{1}{\sqrt{a^{2} r^{2}+b^{2}}} t\right), b \frac{1}{\sqrt{a^{2} r^{2}+b^{2}}} \cos \left(\frac{1}{\sqrt{a^{2} r^{2}+b^{2}}} t\right)\right)
\end{aligned}
$$

i.e., $\mathrm{H}^{2}$ is a regular curve in $\mathrm{E}_{1}^{4}$. Since:

$$
\begin{aligned}
& \mathrm{H}^{2}=\left(-a \frac{r^{2}}{\sqrt{a^{2} r^{2}+b^{2}}} \cos \left(\frac{r}{\sqrt{a^{2} r^{2}+b^{2}}} t\right),\right. \\
& -a \frac{r^{2}}{\sqrt{a^{2} r^{2}+b^{2}}} \sin \left(\frac{r}{\sqrt{a^{2} r^{2}+b^{2}}} t^{*}\right),-b \frac{1}{a^{2} r^{2}+b^{2}} \\
& \cos \left(\frac{1}{\sqrt{a^{2} r^{2}+b^{2}}} t^{*}\right),-b \frac{1}{a^{2} r^{2}+b^{2}} \sin \left(\frac{1}{\sqrt{a^{2} r^{2}+b^{2}}} t^{*}\right),
\end{aligned}
$$

Then the curvature of the helix $K \neq 0$.

Now, we discuss the retractions of the open hyperhelix $\mathrm{H}^{2}$. Let $\mathrm{r}^{\mathrm{i}}$ : $\mathrm{H}^{2} \rightarrow \overline{\mathrm{H}^{2}}, \overline{\mathrm{H}^{2}} \subset \mathrm{H}^{2}$ be the retraction map of $\mathrm{H}^{2}$ such that: $\mathrm{r}_{1}\left(\mathrm{H}^{2}\right)=(\mathrm{a}, 0, \mathrm{~b}, 0),, \mathrm{t}=$ 0 . In this case $r^{\prime}{ }_{1}\left(\mathrm{H}^{2}\right)=0$ and $\left(\mathrm{r}_{1}{ }_{1}, \mathrm{r}_{1}{ }_{1}\right)=0$ then this retraction is a space like curve:

$$
\begin{aligned}
& \mathrm{r}_{2}\left(\mathrm{H}^{2}\right)=\left(\mathrm{a} \cos \left(\frac{\pi \mathrm{r}}{6}\right), \mathrm{a} \sin \left(\frac{\pi \mathrm{r}}{6}\right) \frac{\sqrt{3 \mathrm{~b}}}{2}, \frac{\mathrm{b}}{2}\right), \mathrm{t}=\frac{\pi}{6} \sqrt{\mathrm{a}^{2} \mathrm{r}^{2}+\mathrm{b}^{2}} \\
& \mathrm{r}_{3}\left(\mathrm{H}^{2}\right)=\left(\frac{\sqrt{3 \mathrm{~b}}}{2}\left(\frac{\mathrm{a}}{2}\right), \mathrm{b} \cos \left(\frac{\pi}{6 \mathrm{r}}\right), \mathrm{b} \sin \left(\frac{\pi}{6 \mathrm{r}}\right)\right), \mathrm{t}=\frac{\pi}{6} \sqrt{\mathrm{a}^{2} \mathrm{r}^{2}+\mathrm{b}^{2}} \\
& \mathrm{r}_{4}\left(\mathrm{H}^{2}\right)=\left(\mathrm{a} \cos \left(\frac{\pi \mathrm{r}}{4}\right), \mathrm{a} \sin \left(\frac{\pi \mathrm{r}}{4}\right), \mathrm{a} \sin \left(\frac{\pi \mathrm{r}}{4}\right), \frac{\sqrt{2 \mathrm{~b}}}{2}, \frac{\mathrm{b}}{2}\right), \mathrm{t}=\frac{\pi}{4} \sqrt{\mathrm{a}^{2} \mathrm{r}^{2}+\mathrm{b}^{2}} \\
& \mathrm{r}_{5}\left(\mathrm{H}^{2}\right)=\left(\frac{\sqrt{3 \mathrm{a}}}{2} \frac{\sqrt{2 \mathrm{a}}}{2}, \mathrm{~b} \cos \left(\frac{\pi}{4 \mathrm{r}}\right), \mathrm{b} \sin \left(\frac{\pi}{4 \mathrm{r}}\right)\right), \mathrm{t}=\frac{\pi}{6} \sqrt{\mathrm{a}^{2} \mathrm{r}^{2}+\mathrm{b}^{2}} \\
& \mathrm{r}_{6}\left(\mathrm{H}^{2}\right)=\left(\mathrm{a} \cos \left(\frac{\pi \mathrm{r}}{3}\right), \mathrm{a} \sin \left(\frac{\pi \mathrm{r}}{3}\right), \frac{\mathrm{b}}{2}, \frac{\sqrt{3 \mathrm{~b}}}{2}\right), \mathrm{t}=\sqrt{\mathrm{a}^{2} \mathrm{r}^{2}+\mathrm{b}^{2}}, \\
& \mathrm{r}_{8}\left(\mathrm{H}^{2}\right)=\left(\mathrm{a} \cos \left(\frac{\pi \mathrm{r}}{2}\right), \mathrm{a} \sin \left(\frac{\pi \mathrm{r}}{3}\right), \frac{\sqrt{\pi \mathrm{r}}}{2}, 0, \mathrm{~b} \cos \left(\frac{\pi}{3 \mathrm{r}}\right), \mathrm{b} \sin \left(\frac{\pi}{3 \mathrm{r}}\right)\right), \mathrm{t}=\frac{\pi}{3 \mathrm{r}} \sqrt{\mathrm{a}^{2} \mathrm{r}^{2}+\mathrm{b}^{2}} \\
& \mathrm{r}_{9}\left(\mathrm{H}^{2}\right)=\left(0, \mathrm{a}, \mathrm{b} \cos \left(\frac{\pi}{2}\right), \mathrm{b} \sin \left(\frac{\pi}{2}\right)\right), \mathrm{t}=\frac{\pi}{2 \mathrm{r}} \sqrt{\mathrm{a}^{2} \mathrm{r}^{2}+\mathrm{b}^{2}}, \\
& \mathrm{r}_{11}\left(\mathrm{H}^{2}\right)=\left(-\frac{\sqrt{3 \mathrm{a}}}{2}, \frac{\mathrm{a}}{2}, \mathrm{~b} \cos \left(\frac{5 \pi}{6 \mathrm{r}}\right), \mathrm{b} \sin \left(\frac{5 \pi}{6 \mathrm{r}}\right)\right), \mathrm{t}=\frac{5 \pi}{6 \mathrm{r}} \sqrt{\mathrm{a}^{2} \mathrm{r}^{2}+\mathrm{b}^{2}}, \\
& \mathrm{r}_{10}\left(\mathrm{H}^{2}\right)=\left(\mathrm{a} \cos \left(\frac{5 \pi \mathrm{r}}{6}\right), \mathrm{asin}\left(\frac{5 \pi \mathrm{r}}{6}\right),-\frac{\sqrt{3 \mathrm{~b}}}{2}, \frac{\mathrm{b}}{2}\right), \mathrm{t}=\frac{5 \pi}{6} \sqrt{\mathrm{a}^{2} \mathrm{r}^{2}+\mathrm{b}^{2}}
\end{aligned}
$$

In this retraction $\mathrm{r}_{12}^{\prime}\left(\mathrm{H}^{2}\right)=0$ and $\left(\mathrm{r}_{12}{ }_{12}, \mathrm{r}_{12}^{\prime}\right)=0$, then this retraction is a space like curve. $\mathrm{r}_{13}\left(\mathrm{H}^{2}\right)=(-\mathrm{a}, 0, \mathrm{~b}$ $\left.\cos \left(\frac{\pi}{r}\right), b \sin \left(\frac{\pi}{r}\right)\right), t=\frac{\pi}{r} \sqrt{a^{2} r^{2}+b^{2}}$. In this case $r_{13}{ }_{13}$ $\left(\mathrm{H}^{2}\right)=0$ and $\left(\mathrm{r}_{13}^{\prime}, \mathrm{r}_{13}^{\prime}\right)=0$, then this retraction is a space like curve:

$$
\begin{aligned}
& \mathrm{r}_{14}\left(\mathrm{H}^{2}\right)=\left(\mathrm{a} \cos \left(\frac{5 \pi \mathrm{r}}{4}\right), \mathrm{a} \sin \left(\frac{5 \pi \mathrm{r}}{4}\right),-\frac{\sqrt{2 \mathrm{~b}}}{2},-\frac{\sqrt{\mathrm{b} 2}}{2}\right), \mathrm{t}=\frac{5 \pi}{4} \sqrt{\mathrm{a}^{2} \mathrm{r}^{2}+\mathrm{b}^{2}}, \\
& \mathrm{r}_{15}\left(\mathrm{H}^{2}\right)=\left(-\frac{\sqrt{2 \mathrm{a}}}{2}, \frac{\sqrt{2 \mathrm{a}}}{2}, \mathrm{~b} \cos \left(\frac{5 \pi}{4 \mathrm{r}}\right), \mathrm{b} \sin \left(\frac{5 \pi}{4 \mathrm{r}}\right)\right), \mathrm{t}=\frac{5 \pi}{4 \mathrm{r}} \sqrt{\mathrm{a}^{2} \mathrm{r}^{2}+\mathrm{b}^{2}} \\
& \mathrm{r}_{16}\left(\mathrm{H}^{2}\right)=\left(\mathrm{a} \cos \left(\frac{4 \pi \mathrm{r}}{3}\right), \mathrm{a} \sin \left(\frac{4 \pi \mathrm{r}}{4}\right),-\frac{\mathrm{b}}{2},-\frac{\sqrt{3 \mathrm{~b}}}{2}\right), \mathrm{t}=\frac{4 \pi}{3} \sqrt{\mathrm{a}^{2} \mathrm{r}^{2}+\mathrm{b}^{2}}
\end{aligned}
$$

In this retraction $\mathrm{r}_{16}{ }_{16}\left(\mathrm{H}^{2}\right)=0$ and $\left(\mathrm{r}_{16}{ }_{16}, \mathrm{r}_{16}{ }_{16}\right)=0$, then this retraction is a space like curve: 


$$
\begin{gathered}
\mathrm{r}_{17}\left(\mathrm{H}^{2}\right)=\left(-\frac{\mathrm{a}}{2},-\frac{\sqrt{3 \mathrm{a}}}{2}, \mathrm{~b} \cos \left(\frac{4 \pi}{3 \mathrm{r}}\right), \mathrm{b} \sin \left(\frac{4 \pi}{3}\right)\right), \\
\mathrm{t}=\frac{4 \pi}{3 \mathrm{r}} \sqrt{\mathrm{a}^{2} \mathrm{r}^{2}+\mathrm{b}^{2}}, \mathrm{r}_{18}\left(\mathrm{H}^{2}\right)=\left(\mathrm{a} \cos \left(\frac{3 \pi \mathrm{r}}{2}\right) \mathrm{a} \sin \left(\frac{3 \pi \mathrm{r}}{2}\right), 0,-\mathrm{b}\right), \\
\mathrm{t}=\frac{3 \pi}{2} \sqrt{\mathrm{a}^{2} \mathrm{r}^{2}+\mathrm{b}^{2}}, \mathrm{r}_{19}\left(\mathrm{H}^{2}\right)=\left(0,-\mathrm{a}, \mathrm{b} \cos \left(\frac{3 \pi}{2 \mathrm{r}}\right) \mathrm{b} \sin \left(\frac{3 \pi}{2 \mathrm{r}}\right)\right), \\
\mathrm{t}=\frac{3 \pi}{2 \mathrm{r}} \sqrt{\mathrm{a}^{2} \mathrm{r}^{2}+\mathrm{b}^{2}}, \mathrm{r}_{20}\left(\mathrm{H}^{2}\right)=(0,0,-\mathrm{b}, 0), \mathrm{a}=0, \mathrm{t}=\mathrm{b} \pi, \\
\mathrm{r}_{22}\left(\mathrm{H}^{2}\right)=\left(\mathrm{a}, 0, \mathrm{~b} \cos \left(\frac{\mathrm{t}^{*}}{\mathrm{~b}}\right), \mathrm{b} \sin \left(\frac{\mathrm{t}^{2}}{\mathrm{~b}}\right)=(-\mathrm{a}, 0,0,0), \mathrm{b}=, \mathrm{t}=\mathrm{a} \pi, \mathrm{r}=0, \mathrm{c} \leq \mathrm{t}^{*} \leq \mathrm{c}+1, \mathrm{c} \in \mathbb{R},\right. \\
\mathrm{r}_{23}\left(\mathrm{H}^{2}\right)=\left(\mathrm{a} \cos \left(\frac{\mathrm{t}^{*}}{\mathrm{a}}\right), \mathrm{a} \sin \left(\frac{\mathrm{t}^{*}}{\mathrm{a}}\right), 0,0\right), \mathrm{b}=0, \mid \mathrm{c} \leq \mathrm{t}^{*} \leq \mathrm{c}^{2}, \mathrm{c} \in \mathbb{R},
\end{gathered}
$$

In this case $\mathrm{r}_{23}^{\prime}\left(\mathrm{H}^{2}\right)=\left(-\sin \left(\frac{\mathrm{t}^{*}}{\mathrm{a}}\right), \cos \left(\frac{\mathrm{t}^{*}}{\mathrm{a}}\right), 0,0\right)$ and $\left(r_{23}^{\prime}, r_{23}^{\prime}\right)=1$, then this retraction is a space like curve:

$$
\begin{aligned}
& r_{24}\left(H^{2}\right)=\left(a \cos \left(\frac{r}{\sqrt{a^{2} r^{2}+b^{2}}} t^{*}\right),\right. \\
& a \sin ^{2}\left(\frac{r}{\sqrt{a^{2} r^{2}+b^{2}}} t^{*}\right), b \cos \left(\frac{r}{\sqrt{a^{2} r^{2}+b^{2}}} t^{*}\right), \\
& \left.b \sin \left(\frac{1}{\sqrt{a^{2} r^{2}+b^{2}}} t^{*}\right)\right), \frac{c}{n} \leq t^{*} \leq|c|, c \in R, n \in N \\
& r_{25}\left(H^{2}\right)=\left(a \cos \left(\frac{r}{\sqrt{a^{2} r^{2}+b^{2}}} t^{*}\right),\right. \\
& a \sin \left(\frac{r}{\sqrt{a^{2} r^{2}+b^{2}}} t^{*}\right), b \cos \left(\frac{r}{\sqrt{a^{2} r^{2}+b^{2}}} t^{*}\right), \\
& \left.b \sin \left(\frac{1}{\sqrt{a^{2} r^{2}+b^{2}}} t^{*}\right)\right), c \leq t^{*} \leq n \cdot|c|, c \in R, n \in N \\
& r_{26}\left(H^{2}\right)=\left(a \cos \left(\frac{r}{\sqrt{a^{2} r^{2}+b^{2}}} t^{*}\right),\right. \\
& a \sin \left(\frac{r}{\sqrt{a^{2} r^{2}+b^{2}}} t^{*}\right), b \cos \left(\frac{1}{\sqrt{a^{2} r^{2}+b^{2}}} t^{*}\right), \\
& b \sin \left(\frac{1}{\sqrt{a^{2} r^{2}+b^{2}}} t^{*}\right), c \leq t^{*} \leq d, c<d, c, d \in R
\end{aligned}
$$

In this retraction:

$$
\begin{aligned}
& \mathrm{r}_{26}^{\prime}\left(\mathrm{H}^{2}\right)=\left(-\mathrm{a} \frac{\mathrm{r}}{\sqrt{\mathrm{a}^{2} \mathrm{r}^{2}+\mathrm{b}^{2}}} \sin \left(\frac{\mathrm{r}}{\sqrt{\mathrm{a}^{2} \mathrm{r}^{2}+\mathrm{b}^{2}}} \mathrm{t}^{*}\right),\right. \\
& \mathrm{a} \frac{\mathrm{r}}{\sqrt{\mathrm{a}^{2} \mathrm{r}^{2}+\mathrm{b}^{2}}} \cos \left(\frac{\mathrm{r}}{\sqrt{\mathrm{a}^{2} \mathrm{r}^{2}+\mathrm{b}^{2}}} \mathrm{t}^{*}\right),-\mathrm{b} \frac{1}{\sqrt{\mathrm{a}^{2} \mathrm{r}^{2}+\mathrm{b}^{2}}} \\
& \left.\sin \left(\frac{1}{\sqrt{\mathrm{a}^{2} \mathrm{r}^{2}+\mathrm{b}^{2}}} \mathrm{t}^{*}\right), \mathrm{b} \frac{1}{\sqrt{\mathrm{a}^{2} \mathrm{r}^{2}+\mathrm{b}^{2}}} \cos \left(\frac{1}{\sqrt{\mathrm{a}^{2} \mathrm{r}^{2}+\mathrm{b}^{2}}} \mathrm{t}^{*}\right)\right)
\end{aligned}
$$

And:

$$
\begin{aligned}
& \left(r_{26}, r_{26}\right)=\frac{a^{2} r^{2}}{a^{2} r^{2}+b^{2}}+\frac{b^{2}}{a^{2} r^{2}+b^{2}} \\
& \sin ^{2}\left(\frac{1}{\sqrt{a^{2} r^{2}+b^{2}}} t^{*}\right)-\frac{b^{2}}{a^{2} r^{2}+b^{2}} \cos ^{2}\left(\frac{1}{\sqrt{a^{2} r^{2}+b^{2}}} t^{*}\right) .
\end{aligned}
$$

Then this retraction is a space like curve if:

$$
\begin{aligned}
& \frac{a^{2} r^{2}}{a^{2} r^{2}+b^{2}}+\frac{b^{2}}{a^{2} r^{2}+b^{2}} \\
& \sin ^{2}\left(\frac{1}{\sqrt{a^{2} r^{2}+b^{2}}} t^{*}\right)>\frac{b^{2}}{a^{2} r^{2}+b^{2}} \cos ^{2}\left(\frac{1}{\sqrt{a^{2} r^{2}+b^{2}}} t^{*}\right),
\end{aligned}
$$

a time like curve if:

$$
\begin{aligned}
& \frac{a^{2} r^{2}}{a^{2} r^{2}+b^{2}}+\frac{b^{2}}{a^{2} r^{2}+b^{2}} \\
& \sin ^{2}\left(\frac{1}{\sqrt{a^{2} r^{2}+b^{2}}} t^{*}\right)<\frac{b^{2}}{a^{2} r^{2}+b^{2}} \cos ^{2}\left(\frac{1}{\sqrt{a^{2} r^{2}+b^{2}}} t^{*}\right)
\end{aligned}
$$

and a null like curve if:

$$
\begin{aligned}
& \frac{a^{2} r^{2}}{a^{2} r^{2}+b^{2}}+\frac{b^{2}}{a^{2} r^{2}+b^{2}} \\
& \sin ^{2}\left(\frac{1}{\sqrt{a^{2} r^{2}+b^{2}}} t^{*}\right)=\frac{b^{2}}{a^{2} r^{2}+b^{2}} \cos ^{2}\left(\frac{1}{\sqrt{a^{2} r^{2}+b^{2}}} t^{*}\right) .
\end{aligned}
$$

Hence, we can formulate the following theorems:

Theorem 12: Let $r\left(\mathrm{H}^{2}\right)$ be the retractions map of the hyperhelix $\mathrm{H}^{2} \subset \mathrm{E}_{1}^{4}$. If $\operatorname{dim} \mathrm{r}\left(\mathrm{H}^{2}\right)=0$, then $\mathrm{T}, \mathrm{N}, \mathrm{B}, \mathrm{K}$ and $\tau$ of the retractions of hyperhelix are unlimited. If $\operatorname{dim} r\left(\mathrm{H}^{2}\right)=\operatorname{dim} \mathrm{H}^{2}$, then $\mathrm{T}, \mathrm{N}, \mathrm{B}, \mathrm{K}$ and $\tau$ of $\mathrm{r}\left(\mathrm{H}^{2}\right)$ are the same as or different from $\mathrm{T}, \mathrm{N}, \mathrm{B}, \mathrm{K}$ and $\tau$ of $\mathrm{H}^{2}$.

Theorem 13: The retractions of the hyperhelix $\mathrm{H}^{2} \subset \mathrm{E}_{1}^{4}$ are retractions which preserve the dimension and retractions which do not preserve the dimension.

Theorem 14: Let $r\left(\mathrm{H}^{2}\right)$ be the retraction map of the hyperhelix $\mathrm{H}^{2} \subset \mathrm{E}_{1}^{4}$. If $\operatorname{dim} \mathrm{r}\left(\mathrm{H}^{2}\right)=0$, then this 
retraction of the hyperhelix is a space like curve. If dim $\mathrm{r}\left(\mathrm{H}^{2}\right)=\operatorname{dim} \mathrm{H}^{2}$, then this retraction of the hyperhelix is a space like curve if:

$$
\begin{aligned}
& \frac{a^{2} r^{2}}{a^{2} r^{2}+b^{2}}+\frac{b^{2}}{a^{2} r^{2}+b^{2}} \sin ^{2}\left(\frac{1}{\sqrt{a^{2} r^{2}+b^{2}}} t^{*}\right) \\
& >\frac{b^{2}}{a^{2} r^{2}+b^{2}} \cos ^{2}\left(\frac{1}{\sqrt{a^{2} r^{2}+b^{2}}} t^{*}\right),
\end{aligned}
$$

a time like curve if:

$$
\begin{aligned}
& \frac{a^{2} r^{2}}{a^{2} r^{2}+b^{2}}+\frac{b^{2}}{a^{2} r^{2}+b^{2}} \sin ^{2}\left(\frac{1}{\sqrt{a^{2} r^{2}+b^{2}}} t^{*}\right) \\
& <\frac{b^{2}}{a^{2} r^{2}+b^{2}} \cos ^{2}\left(\frac{1}{\sqrt{a^{2} r^{2}+b^{2}}} t^{*}\right),
\end{aligned}
$$

or a null like curve if:

$$
\begin{aligned}
& \frac{a^{2} r^{2}}{a^{2} r^{2}+b^{2}}+\frac{b^{2}}{a^{2} r^{2}+b^{2}} \sin ^{2}\left(\frac{1}{\sqrt{a^{2} r^{2}+b^{2}}} t^{*}\right) \\
& =\frac{b^{2}}{a^{2} r^{2}+b^{2}} \cos ^{2}\left(\frac{1}{\sqrt{a^{2} r^{2}+b^{2}}} t^{*}\right)
\end{aligned}
$$

Theorem 15: If the deformations retract of the hyperhelix $\mathrm{H}^{2} \subset \mathrm{E}_{1}^{4}$ is $\mathrm{D}: \mathrm{H}^{2} \times \mathrm{I} \rightarrow \mathrm{H}^{2}$ where $\mathrm{I}$ is the closed interval $[0,1]$, the retraction of $\mathrm{H}^{2} \subset \mathrm{E}_{1}^{4}$ is $\mathrm{r}: \mathrm{H}^{2} \rightarrow \mathrm{H}^{1}$, $\mathrm{H}^{1} \subset \mathrm{H}^{2}$ and the limit of the folding of $\mathrm{H}^{2}$ is $\lim _{\mathrm{m} \rightarrow \infty} \mathrm{r}_{\mathrm{m}}$ : $\mathrm{H}^{2} \rightarrow \mathrm{H}^{1}$. Then there induce deformation retract, retractions and the limit of the foldings such that the following diagram is commutative.

Proof: Let the deformation retract of $\mathrm{H}^{2} \subset \mathrm{E}_{1}^{4}$ is $\mathrm{D}_{1}$ : $\mathrm{H}^{2} \times \mathrm{I} \rightarrow \mathrm{H}^{2}$, the retraction of $\mathrm{H}^{2} \times \mathrm{I}$ is defined by $\mathrm{r}_{1}$ : $\left(H^{2} \times I\right) \rightarrow \quad H^{1} \times I, \quad \lim _{m \rightarrow \infty} \quad f_{m} \quad: D_{1} \quad\left(H^{2} \times I\right) \rightarrow H^{1}, \quad$ the deformation retract of $\mathrm{r}_{1}\left(\mathrm{H}^{2} \times \mathrm{I}\right)$ isD $\mathrm{D}_{2}: \mathrm{r}_{1}\left(\mathrm{H}^{2} \times \mathrm{I}\right) \rightarrow \mathrm{H}^{1}$, the retraction of $\lim _{m \rightarrow \infty} f_{m}\left(D_{1}\left(\left(H^{2} \times I\right)\right)\right)$ is given by $r_{2}$ : $\lim _{m \rightarrow \infty} f_{m} \quad\left(D_{1}\left(H^{2} \times I\right)\right) \rightarrow H^{0}$ and $\lim _{m \rightarrow \infty} f_{m+1}: D_{2}$ $\left(\mathrm{r}_{1}\left(\mathrm{H}^{2} \times \mathrm{I}\right) \rightarrow \mathrm{H}^{0}, \mathrm{H}^{0}\right.$ is a 0 -dimensional space. Hence, the following diagram is commutative:

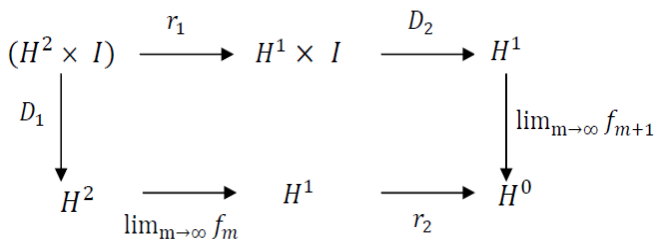

i. e., $\lim _{\mathrm{m} \rightarrow \infty} f_{\mathrm{m}+1}$ o $\mathrm{D}_{2}$ o $\mathrm{r}_{1}\left(\mathrm{H}^{2} \times \mathrm{I}\right)=\mathrm{r}_{2}$ o $\lim _{\mathrm{m} \rightarrow \infty} f_{\mathrm{m}} \mathrm{o}$ $\mathrm{D}_{1}\left(\mathrm{H}^{1} \times \mathrm{I}\right)$
Theorem 16: Let $\mathrm{H}^{2} \subset \mathrm{E}_{1}^{4}$ be the hyperhelix in 4Minkowski space, then the relation between the folding $\mathrm{f}$ : $\mathrm{H}^{2} \rightarrow \mathrm{H}^{2}$ and the limit of the retractions $\lim _{\mathrm{m} \rightarrow \infty} \mathrm{r}_{\mathrm{m}}: \mathrm{H}^{2} \rightarrow$ $\mathrm{H}^{1}$, discussed from the following commutative diagram.

Proof: Let the folding be $f_{1}: \mathrm{H}^{2} \rightarrow \mathrm{H}^{2}$, the limit of the retractions of $\mathrm{H}^{2}$ and $f_{1}\left(\mathrm{H}^{2}\right)$ are $\lim _{\mathrm{m} \rightarrow \infty} \mathrm{r}_{\mathrm{m}}: \mathrm{H}^{2} \rightarrow \mathrm{H}^{1}$ and $\lim _{\mathrm{m} \rightarrow \infty} \mathrm{r}_{\mathrm{m}+1}: f_{1}\left(\mathrm{H}^{2}\right) \rightarrow \mathrm{H}^{1}$ and $f_{2}:\left(\lim _{\mathrm{m} \rightarrow \infty} \mathrm{r}_{\mathrm{m}}\left(\mathrm{H}^{2}\right)\right) \rightarrow \mathrm{H}^{1}$. Then, the following commutative diagram exists:

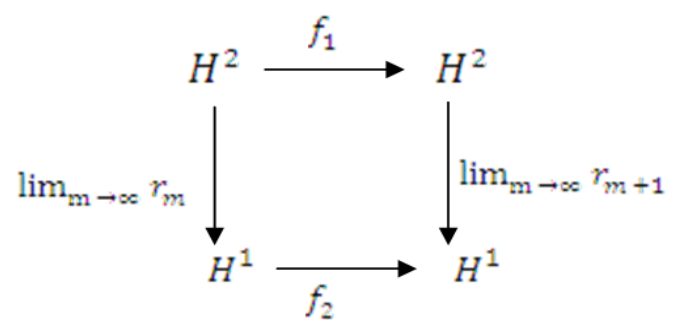

i. e., $\lim _{\mathrm{m} \rightarrow \infty} \mathrm{r}_{\mathrm{m}+1}$ o $f_{1}\left(\mathrm{H}^{2}\right)=f_{2} \circ \lim _{\mathrm{m} \rightarrow \infty} \mathrm{r}_{\mathrm{m}}\left(\mathrm{H}^{2}\right)$.

Theorem 17: Let the retraction of $\mathrm{H}^{2}$ is $\mathrm{r}: \mathrm{H}^{2} \rightarrow \mathrm{H}^{1}, \mathrm{H}^{1} \subset$ $\mathrm{H}^{2}$ and the folding of $\mathrm{H}^{2}$ is $f: \mathrm{H}^{2} \rightarrow \mathrm{H}^{2}$, then

- $f_{2} \circ \mathrm{r}_{1}\left(\mathrm{H}^{2}\right)=\mathrm{r}_{2} \circ f_{1}\left(\mathrm{H}^{2}\right)$

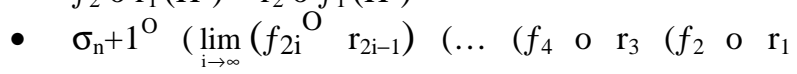
$\left.\left.\left.\left.\left(\mathrm{H}^{2}\right)\right)\right) \ldots\right)\right)=\left(\lim _{\mathrm{i} \rightarrow \infty}\left(f_{2 \mathrm{i}}{ }^{\mathrm{O}} \mathrm{r}_{2 \mathrm{i}-1}\right)\left(\ldots\left(\mathrm{r}_{4} \circ f_{3}\left(\mathrm{r}_{2} \circ f_{1}\right.\right.\right.\right.$ $\left.\left.\left.\left.\left(\mathrm{H}^{2}\right)\right)\right) \ldots\right)\right)$ о $\sigma_{1}$

Proof: Let the retraction of the hyperhelix in 4Minkowski space $\mathrm{H}^{2}$ is $\mathrm{r}_{1}: \mathrm{H}^{2} \rightarrow \mathrm{H}^{1}, f_{1}: \mathrm{H}^{2} \rightarrow \mathrm{H}^{2}$, the retraction of $f_{1}\left(\mathrm{H}^{2}\right)$ is $\mathrm{r}_{2}: f_{1}\left(\mathrm{H}^{2}\right) \rightarrow \mathrm{H}^{1}$ and the folding of $\mathrm{r}_{1}$ $\left(\mathrm{H}^{2}\right)$ is $f_{2}: \mathrm{r}_{1}\left(\mathrm{H}^{2}\right) \rightarrow \mathrm{H}^{1}$. Then $f_{2}$ o $\mathrm{r}_{1}\left(\mathrm{H}^{2}\right)=\mathrm{r}_{2}$ o $f_{1}\left(\mathrm{H}^{2}\right)$.

Let $f_{2 \mathrm{i}} \quad \mathrm{O} \mathrm{r}_{2 \mathrm{i}}-1$ and $\mathrm{r}_{2 \mathrm{i}} \circ \mathrm{f}_{2 \mathrm{i}}-1$ are the compositions between the retractions of the hyperhelix in 4Minkowski space $\mathrm{H}^{2}$ and the foldings of $\mathrm{H}^{2}$ into itself. Also, $\sigma_{\mathrm{I}}$ are the homeomorphisms. Then:

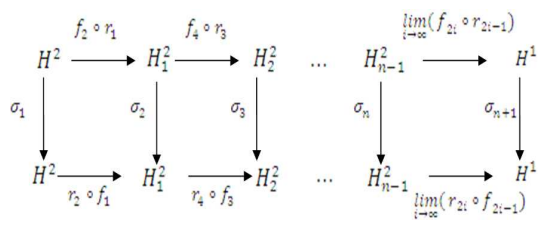

Theorem 18: Given the deformation retract of $\mathrm{H}^{2} \subset$ $\mathrm{E}_{1}^{4}$ is $\mathrm{D}: \mathrm{H}^{2} \times \mathrm{I} \rightarrow \mathrm{H}^{2}$, the limit of the folding of $\mathrm{H}^{2} \times \mathrm{I}$ is $\lim _{\mathrm{m} \rightarrow \infty} f_{\mathrm{m}} \mathrm{H}^{2} \times \mathrm{I} \rightarrow \mathrm{H}^{1} \times \mathrm{I}$. Then, the following diagram is commutative.

Proof: Let the limit of the folding of $\left(\mathrm{H}^{2} \times \mathrm{I}\right)$ is $\lim _{\mathrm{m} \rightarrow \infty} f_{\mathrm{m}}: \mathrm{H}^{2} \times \mathrm{I} \rightarrow \mathrm{H}^{2} \times \mathrm{I}$, the deformation retract of $\mathrm{H}^{2} \subset \mathrm{E}_{1}^{4}$ is $\mathrm{D}_{1}: \mathrm{H}^{2} \times \mathrm{I} \rightarrow \mathrm{H}^{2}$, the limit of the folding of 
$\mathrm{D}_{1}\left(\mathrm{H}^{2} \times \mathrm{I}\right)$ is $\lim _{\mathrm{m} \rightarrow \infty} f_{\mathrm{m}+1}: \mathrm{D}_{1}\left(\mathrm{H}^{2} \times \mathrm{I}\right) \rightarrow \mathrm{H}^{1}$ and the deformation retract of $\lim _{\mathrm{m} \rightarrow \infty} \mathrm{f}_{\mathrm{m}}\left(\mathrm{H}^{2} \times \mathrm{I}\right)$ is $\mathrm{D}_{2}$ : $\lim _{\mathrm{m} \rightarrow \infty} f_{\mathrm{m}}\left(\mathrm{H}^{2} \times \mathrm{I}\right) \rightarrow \mathrm{H}^{1}$. Hence:

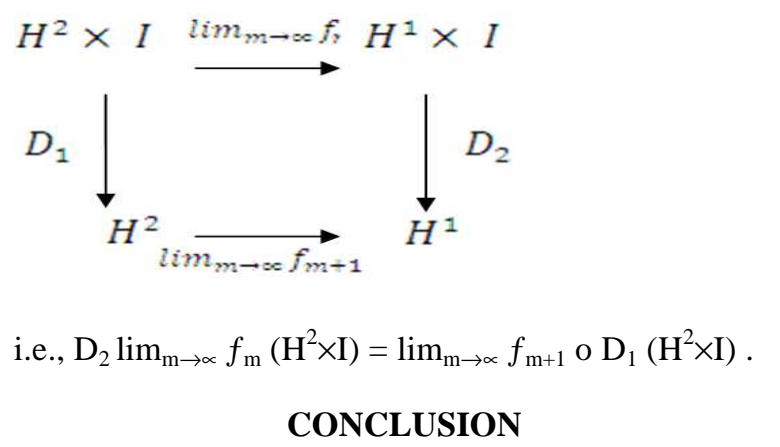

In this study we achieved the approval of the importance of the retractions of the hyperhelix in Minkowski space. The relations between foldings, retractions and deformation retract, limits of folding and the limits of retractions of hyperhelix in Minkowski space are discussed. A theorem which governs these relations is presented.

\section{REFERENCES}

Baronti, M., E. Casini and C. Franchetti, 2003. The retraction constant in some Banach spaces. J. Approximation Theory, 120: 296-308. DOI: 10.1016/S0021-9045 (02)00037-0

Catoni, F., D. Boccaletti, R. Cannata, V. Catoni and E. Nichelatti et al., 2008. The Mathematics of Minkowski Space-Birkhauser Verlag. 1st Edn., Basel, Boston, Berlin.

Demainel, E.D., 2001. Folding and Unfolding, Ph.D. Thesis, Waterloo University, Canada.

Di-Francesco, P., 2000. Folding and coloring problems in mathematics and physics. Bulliten Am. Math. Soc., 37: 251-307.

El-Ahmady, A.E. and H. Rafat, 2006. A calculation of geodesics in chaotic flat space its folding. Chaos Solutions Fractals, 30: 836-844. DOI: 10.1016/j.chaos.2005.05.033

El-Ahmady, A.E. and H. Rafat, 2009. Retraction of chaotic Ricci space. Chaos Solutions Fractals, 41: 394-400. DOI: 10.1016/j.chaos.2008.01.010

El-Ahmady, A.E. and A. El-Araby, 2010. On fuzzy spheres in fuzzy Minkowski space. Nuovo. Cimento., 125B: 1-8.

El-Ahmady, A.E. and E. Al-Hesiny, 2011. The topological folding of the hyperbola in Minkowski 3-space. Inter. J. Nonlinear Sci., 11: 451-458.

El-Ahmady, A.E. and H.M. Shamara, 2001. Fuzzy deformation retract of fuzzy horospheres. Ind. J. Pure. Appl. Math., 32: 1501-1506.
El-Ahmady, A.E., 1994. The deformation retract and topological folding of Buchdahi space. Periodica Math. Hungarica, 28: 19-30.

El-Ahmady, A.E., 2004a. Fuzzy folding of fuzzy horocycle. Rendiconti del Circolo Matematico di Palermo, 53: 443-450. DOI: 10.1007/BF02875737

El-Ahmady, A.E., 2004b. Fuzzy Lobachevskian space and its folding. J. Fuzzy Math., 12: 609-614.

El-Ahmady, A.E., 2006. Limits of fuzzy retractions of fuzzy hyperspheres and them folding. Tamkang J. Math., 37: 47-55.

El-Ahmady, A.E., 2007a. The variation of the density on chaotic spheres in chaotic space-like Minkowski space time. Chaos Solitons Fractals, 31: 12721278. DOI: $10.1016 /$ j.chaos.2005.10.s112

El-Ahmady, A.E., 2007b. Folding of fuzzy hypertori and their retractions. Proc. Math. Phys. Soc. Egypt., 85: 1-10.

El-Ahmady, A.E., 2011a. Retraction of chaotic black hole. J. Fuzzy Math., 19: 833-838.

El-Ahmady, A.E., 2011b. The geodesic deformation retract of Klein bottle and its folding. Inter. J. Nonlinear Sci., 12: 323-330.

Ilarslan, K. and O. Boyacioglu, 2008. Position vectors of a time like a null helix in Minkowski 3-space. Chaos Solitons Fractals, 38: 1383-1389. DOI: 10.1016/j. chaos. 2008.04.003

Kocayigit, H. and M. Onder, 2007. Time like curves of contest slope in Minkowski space $\mathrm{E}_{1}{ }^{4}$. J. Sci. Technol., 1: 2-2.

Lopez, R., 2008. Differential Geometry of Curves and Surfaces in Lorentz-Minkowski Space. Instituto de Matematica e Estatistica, University of Sao Paulo, Brazil

Michael, E. 2003. Closed retract perfect retracts. Topology Appli. 12: 451-468. DOI: 10.1016/S0166-8641 (01)00208-5

Naber, G.L., 2011a. Topology, Geometry and Gauge fields: Foundations. 2nd Eds., Springer-Verlage New York, Berlin, ISBN-10: 1441972536 pp: 440.

Naber, G.L., 2011b. Topology, Geometry and Gauge fields: Interactions. 2nd Edn., Springer-Verlage New York, ISBN-10: 1441978941 pp: 419.

Pellicer-Covarrubias, P., 2004. Retractions in hyperspaces. Topology Appli., 135: 277-291. DOI: 10.1016/S0166-8641 (03)00193-7

Reid, M. and B. Szendroi. 2005. Topology and Geometry. 1st Edn., Cambridge University Press, Cambridge, New York.

Shick, P., 2007. Topology: Point-Set and Geometry. 1st Edn., John Wiley and Sons, ISBN-10: 0470096055 pp: 271.

Walrave, J., 1995. Curves and surfaces in Minkowski space. Ph.D. Thesis, K.U. Leuven.

Yaliniz, A.F. and H.H. Hacisalihoglu, 2007. Null generalized helices in $\mathrm{L}^{\mathrm{M}+2}$. Bull. Malays. Math. Sci. Soc., 2: 30-174-85. 